\title{
Vibrational phonon spectroscopy of boron nitride polymorphs: a comparison between theory and experiment
}

\author{
R. Nicholls ${ }^{1}$, F.S. Hage ${ }^{2}$, J. Yates ${ }^{1}$, D. McCulloch ${ }^{3}$, D.M. Kepaptsoglou ${ }^{2}$, T.C. Lovejoy ${ }^{4}$, N. Dellby ${ }^{4}$, \\ O.L. Krivanek ${ }^{4}$, K. Refson ${ }^{5,6}$ and Q.M. Ramasse ${ }^{2}$ \\ ${ }^{1}$ Department of Materials, University of Oxford, Parks Road, Oxford OX1 3PH, U.K. \\ 2 SuperSTEM Laboratory, STFC Daresbury Campus, Keckwick Lane, Daresbury WA4 4AD, U.K. \\ ${ }^{3}$ School of Applied Sciences, RMIT University, Melbourne VIC 3001, Australia \\ ${ }^{4}$ Nion Company, 11511 NE $118^{\text {th }}$ St., Kirkland, WA 98034, U.S.A. \\ ${ }^{5}$ STFC Rutherford Appleton Lab., Harwell Science and Innovation Campus, Didcot OX11 0QX, U.K. \\ ${ }^{6}$ Department of Physics, Royal Holloway, University of London, Egham TW20 0EX, U.K.
}

The increased energy resolution of a new generation of electron microscopes has made exploring the phonon region of the EELS spectrum possible [1]. To fully interpret the extra information available it is important to be able to compare the data to theoretical calculations so that we can extract physically meaningful results.

Here we use different polymorphs of boron nitride and in particular the cubic $(c-\mathrm{BN})$ and hexagonal ( $h$ $\mathrm{BN}$ ) phases, as well as other BN-containing complex engineering materials, to compare experimental phonon spectroscopy results in the scanning transmission electron microscope to theoretical calculations. Both cubic and hexagonal boron nitride samples were prepared by simple drop casting onto lacey carbon grids. Imaging and EELS core loss and phonon spectrum imaging were carried out on the different BN polymorphs using a monochromated Nion UltraSTEM100MC 'HERMES' microscope [2] installed at the SuperSTEM Laboratory. Atomic resolution images and core loss chemical maps of the regions of interest were obtained in order to fully characterize them prior to phonon spectroscopy. For the high energy resolution experiments, the instrument was subsequently adjusted to provide a zero-loss peak (ZLP) full width at half-maximum (FWHM) of approximately $15 \mathrm{meV}$ for a probe size of $2.5 \AA$. The microscope was operated at $60 \mathrm{kV}$ in order to minimize any damage to the samples.

Typical phonon spectra are shown in figure 1: all spectra were normalized to the maximum of the zeroloss peak (ZLP) to account for total signal variations as the probe was moved into the rather thick BN flakes. Several very striking features can be observed. For $h$-BN, the main phonon peak appears at $183 \mathrm{meV}$ (note that the difference with previously reported values [1] could be due flake size effects or to slight energy calibration differences). Interestingly, a small energy shift is observed as the probe moves from aloof geometry into the sample. The FWHM of the peak also increases within the sample. By contrast, the main phonon for $c$-BN appears at a lower energy of $147 \mathrm{meV}$. A small shoulder at $\sim 100 \mathrm{meV}$ appears only when the beam goes through the sample: its origin is being investigated.

Phonon modes are a property of the material and several techniques exist to calculate them including finite-displacement methods and perturbation theory. The intensity with which the modes appear in an experimental spectrum depends on the type of experiment and experimental set up. Several $a b$ initio codes are able to compute phonon modes in solids together with IR and Raman intensities. We use the density functional theory code CASTEP [3] to calculate the phonon modes for the different BN polymorphs using a linear response method [4] and compare these to the experimentally observed spectra. One of the in-plane optical modes of $h$-BN is shown in Figure 2. 


\section{References}

[1] O.L. Krivanek, T.C. Lovejoy, N. Dellby et al., Nature 514 (2014), pp. 209-212.

[2] O.L. Krivanek, J.P. Ursin, N.J. Bacon et al., Phil. Trans. Roy. Soc. 367 (2009), pp. 3683-3697.

[3] S.J. Clark, M.D. Segall, C.J. Pickard et al., Z. Kristallog. 220 (2005), pp. 567-570.

[4] K. Refson, S.J. Clark and P.R. Tulip, Phys. Rev. B 73 (2006), 155114.

[5] SuperSTEM is funded by the UK Engineering and Physical Sciences Research Council (EPSRC).
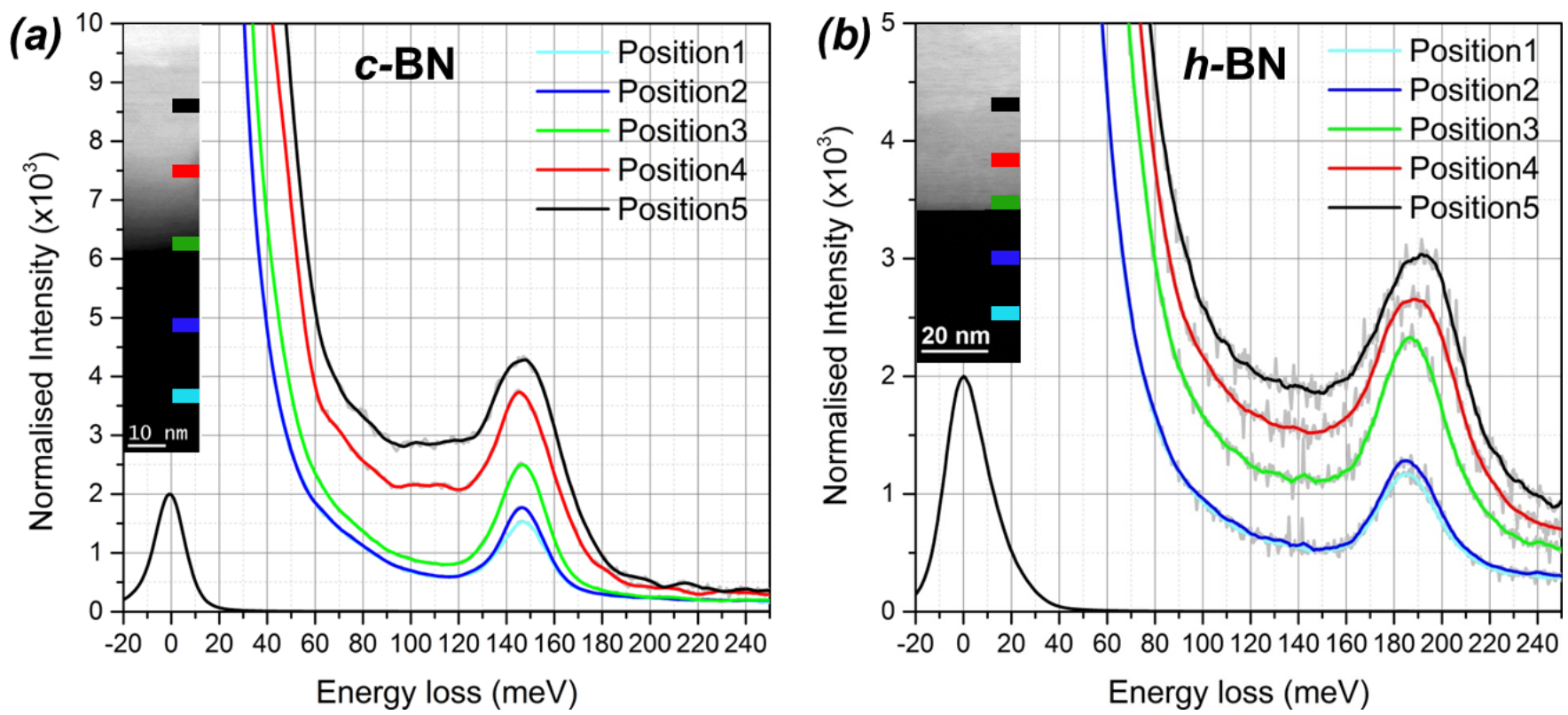

Figure 1. Position-resolved low loss EEL spectra taken at the edge and into (a) $c$-BN and (b) $h$-BN flakes. HAADF images, inset, show the exact location of the EELS acquisition with respect to the flake edge. 100 frames of $8 \mathrm{~ms}$ (resp. 100 frames of 5ms) exposure each were averaged to improve the signalto-noise for the $c$-BN (resp. $h$-BN), after careful alignment to their maximum. The full ZLP is shown for comparison, scaled by a factor of 1000 (a) (resp. 500, (b)).
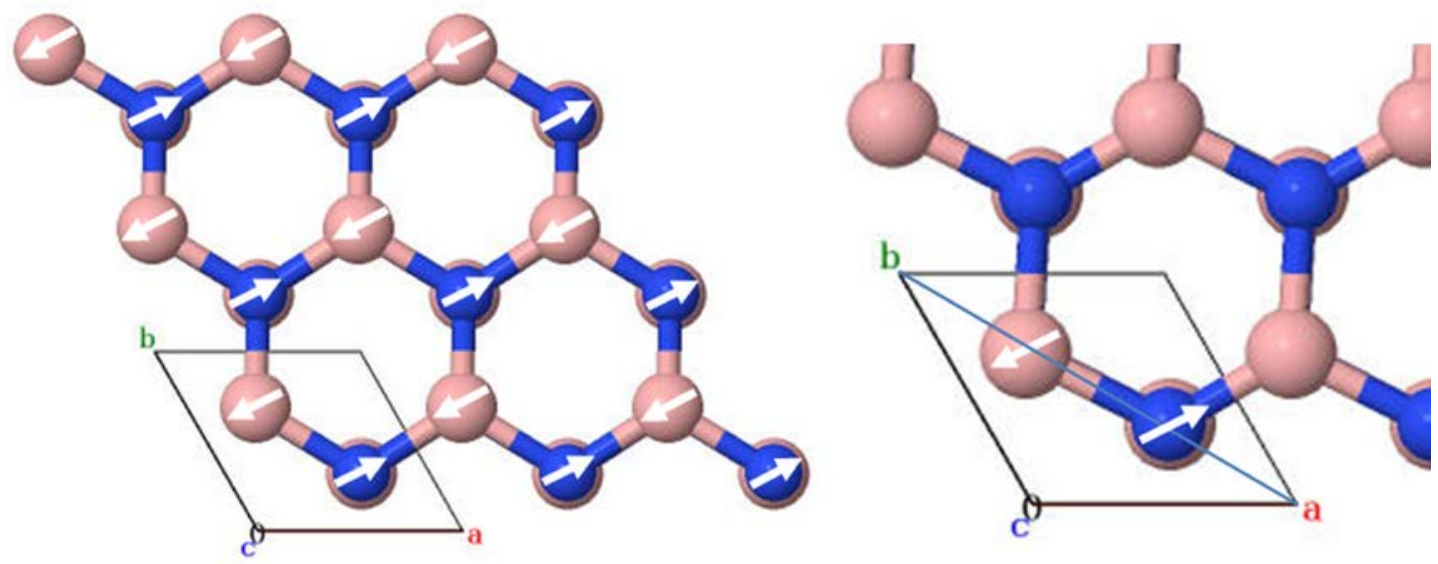

Figure 2. Simulated in-plane optical phonon mode for $h$-BN. Each column of atoms moves as a whole and, within each layer, the carbon-nitrogen bond oscillates. 